\title{
Neoadjuvant Rectal (NAR) Score: a New Surrogate Endpoint in Rectal Cancer Clinical Trials
}

\author{
Thomas J. George Jr. ${ }^{1,3}$ • Carmen J. Allegra ${ }^{1,3}$ • Greg Yothers ${ }^{2,3}$
}

Published online: 9 August 2015

(C) The Author(s) 2015. This article is published with open access at Springerlink.com

\begin{abstract}
The conduct of clinical trials in colorectal cancer has historically relied upon endpoints such as disease-free (DFS) or overall survival (OS). While ideal, these endpoints require longterm follow-up, thus contributing to a slow pace of scientific progress in clinical research. Identification of short-term endpoints to serve as surrogates for DFS and OS would enable more rapid determination of success or failure of an experimental intervention and thus facilitate more scientific discovery and progress leading to clinical practice improvements. In rectal cancer clinical trials, there have been few validated alternatives to DFS and OS, including pathologic complete response (ypCR). The neoadjuvant rectal (NAR) score was developed as a composite short-term endpoint for clinical trials involving neoadjuvant therapy for rectal cancer. The NAR score is based upon variables routinely collected and available to clinical investigators during the conduct of prospective studies. Based upon two independent validation datasets, the NAR score predicts OS in rectal cancer clinical trials better than ypCR. While final dataset validation is
\end{abstract}

Topical Collection on Localized Colorectal Cancer

Support U10-CA-12027, U10-CA-37377, U10-CA-69651, U-10-CA69974; Sanofi; Roche

Thomas J. George, Jr.

thom.george@medicine.ufl.edu

Carmen J. Allegra

carmen.allegra@medicine.ufl.edu

Greg Yothers

YothersG@NRGOncology.org

1 University of Florida Health Cancer Center, Gainesville, 1600 SW Archer Rd, PO Box 100278, Gainesville, FL 32610, USA

2 University of Pittsburgh, One Sterling Plaza, 201 N Craig St, Ste 350, Pittsburgh, PA 15213, USA

3 NRG Oncology, Four Penn Center, 1600 JFK Blvd, Suite 1020, Philadelphia, PA 19103, USA ongoing, the NAR score offers an opportunity to incorporate a novel surrogate endpoint into early phase rectal cancer clinical trials.

Keywords Rectalcancer $\cdot$ NAR score $\cdot$ Surrogate $\cdot$ Endpoint . Pathologic complete response $\cdot$ Clinical trial $\cdot$ Neoadjuvant . Nomogram · pCR

\section{Introduction}

Colorectal cancer (CRC) represents a highly prevalent but treatable cancer. It is estimated that in 2015 , there will be 132,700 new cases of CRC and 49,700 deaths from CRC in the USA alone, accounting for it as the second leading cause of cancer death [1]. The impact of an effective national screening program has been well defined [2•]. With screening compliance improving, rates for new colon and rectum cancer cases have been falling on average by approximately $3 \%$ each year over the last 10 years, but death rates have not changed significantly over 2002-2012 [1]. Rectal cancer, also referred to as a distal large bowel adenocarcinoma within $12-15 \mathrm{~cm}$ from the anal verge, represents a subset of colorectal cancer cases. For rectal cancer in the USA, there will be 39,610 new cases in 2015 (male 23,200; female 16,410) representing nearly $1 / 3$ of the total CRC cases [3].

Despite similar molecular and genetic profiles, rectal cancer is treated differently from colon cancer in several important ways [4••]. First, because of the pelvic anatomy and risk for close surgical margins, rectal cancer management must include consideration for local disease control. This is optimized by performing total mesorectal excision (TME) and perioperative chemoradiotherapy (CRT) [5]. Second, patients with low lying rectal cancer are at risk for loss of sphincter function, requiring permanent colostomy or suffer from fecal incontinence. Finally, 
risk stratification and management of rectal cancer requires multidisciplinary staging and care coordination including pelvic magnetic resonance imaging (MRI) or endoscopic ultrasound (EUS). Non-operative treatment decisions are made without the benefit of pathologic staging since surgical resection is typically delayed until after neoadjuvant therapy.

Improvements in rectal cancer patient care have come as the result of clinical trials testing new treatments and validating hypotheses. These pragmatic trials have provided significant improvements in disease staging, local disease control, patient survival, quality of life, and sphincter preservation [6]. As a result of several recent pivotal trials, there has been a paradigm shift to include fluoropyrimidine-based CRT in the neoadjuvant setting $[7 \bullet \bullet, 8,9 \bullet \bullet$. Consequently, there is a tremendous desire to use the initial clinical and pathologic features or the in vivo treatment effect as a surrogate for longer term outcomes, both for individual patients and also as a validated endpoint for the next generation of clinical trials. Identifying a valid surrogate short-term endpoint would allow determination of treatment efficacy in clinical trials in a shorter period of time, resolving hypotheses and allowing clinical progress to be made in more rapid fashion. Here, we will focus on surrogate endpoints in rectal cancer clinical trials with a newly validated surrogate, the neoadjuvant rectal (NAR) score.

\section{Surrogate Endpoints}

The idea of using a surrogate endpoint (one that occurs earlier or is more easily attainable) in lieu of a final (or true) endpoint is not a new concept in clinical trial design [10]. Guidelines, considerations, and limitations for establishing surrogate endpoints in clinical trials have been proposed by the National Institutes of Health [11]. A surrogate endpoint requires twostep validation through randomized controlled clinical trials to ensure that (1) it correlates with the true endpoint (aka individual level association) and (2) the effects of treatments that impact the surrogate and true endpoints correlate (aka trial level association) [12].

As previously stated, locoregional relapse was initially a primary form of treatment failure in this disease. However, with the introduction of TME and radiotherapy, local failure is far exceeded by systemic metastatic development. The latter significantly influences disease-free (DFS) and overall survival (OS). As such, these two benchmarks have been the primary endpoints of most major rectal cancer clinical trials in the past three decades $[13,14]$. The prolongation of survival as a primary goal of new therapeutic interventions in rectal cancer is laudable, but complicated by several highly effective agents in the metastatic setting, improved supportive care, and opportunities for salvage surgical resection or ablation of oligometastatic disease. Thus, the duration of patient followup from completion of rectal cancer treatment intervention until death, as a primary endpoint, can take perhaps 3 to 5 years longer than the original conduct of the entire clinical trial itself. Although slightly shorter in time as an endpoint, the same is true for DFS. In addition to the two-step validation described above, an ideal surrogate endpoint would be achieved at or near the completion of the clinical trial intervention, be highly reproducible across different clinical trial study designs and interventions, and be accomplished without added complexity or cost to the patient or the clinical trial design while correlating with the true endpoints of DFS and OS (Table 1).

With the widespread use of TME, pathologic standardization has become increasingly critical for accurate assessment of nodal involvement, margin status, and pathologic staging. While sentinel nodes and clinical responses have proved largely to be poorly representative of systemic disease, histopathology assessments have become critically important [15, 16]. However, the histopathologic assessment of the tumor specimen (and thus any associated variable desired as a surrogate endpoint) is highly dependent upon the quality of the pathologist review [17]. For local risk of recurrence, it was verified that the circumferential radial margin status serves as a highly valuable surrogate endpoint, even in the era of TME $[18,19]$. Attempts to identify a surrogate endpoint for DFS and OS have proved more challenging. However, the introduction of neoadjuvant CRT has offered the opportunity to assess the degree of in vivo treatment effect and downstaging as a potential surrogate for longer term outcomes. One popular endpoint, pathologic complete response (ypCR) represents the ultimate degree of tumor downstaging defined as no histopathologic visible residual tumor remaining after neoadjuvant therapy. This endpoint has been extensively studied in phase III randomized controlled trials $[7 \bullet \bullet, 8,9 \bullet \bullet]$. However,

Table 1 Required and optimal parameters for a surrogate endpoint in rectal cancer clinical trials

Required elements Optimal additional elements

Correlates with the true endpoint (individual level association)

Surrogate endpoint achieved at or near completion of the experimental intervention

Effect of treatment correlates between surrogate and true endpoint High reproducibility across different study designs and interventions (trial level association) 
consistent across these studies was the finding that despite increasing the ypCR, which did correlate with improved local control, OS was not significantly impacted. Pathologic complete response after neoadjuvant CRT is dependent upon the inherent chemo-radiosensitivity of the cancer, bulk of the original tumor, and interval after completion of CRT. While ypCR has been suggested to be a valuable trial surrogate, it has not been endorsed as a validated endpoint in part due to these limitations [20-22].

Since ypCR represents a binary "all or nothing" histopathologic variable, a continuum of treatment regression has been proposed to represent treatment response as a surrogate for survival endpoints [23]. Tumor regression grade (TRG) requires standardization, as there are at least four different institutional or programmatic versions currently in use (Table 2) [24•, 25•, 26-29]. All versions remain relatively subjective in pathologic scoring, usually requiring central pathologic review when incorporated into multicenter clinical trials [30-33]. Early studies involving radiographic imaging modalities (such as MRI, contrast-enhanced cross-sectional imaging, and FDG-PET) demonstrate the potential to serve as surrogates for histopathology, with their own limitations on standardization, reproducibility, and generalizability [34-39]. While each of these proposed endpoints fulfills some of the criteria for a surrogate endpoint (Table 1), challenges remain in validation and widespread reproducibility.

\section{Neoadjuvant Rectal Score}

Valentini and colleagues developed a nomogram for predicting local recurrence, distant metastases, and OS for patients with locally advanced rectal cancer [40••]. The nomogram for OS takes into consideration the clinical tumor (cT) stage, pathologic tumor $(\mathrm{pT})$ stage, pathologic nodal $(\mathrm{pN})$ stage, patient age, adjuvant chemotherapy administration, surgery type (APR vs. LAR), dose of radiotherapy, and gender [40••]. The OS nomogram had a very respectable $c$-index $(0.70)$ supporting a strong correlation which was derived from external validation in five European rectal cancer clinical trials [7••, 8, 41-43].

The NAR score was developed as a short-term clinical trial surrogate endpoint to take variables associated with treatment effect beyond ypCR into consideration yet simple enough to support a diversity of clinical trial designs. The NAR score is calculated based on data supported by the Valentini nomogram for OS, but only using the clinical T stage and pathologic $\mathrm{T}$ and $\mathrm{N}$ stages (Fig. 1). Of the eight variables used in the Valentini nomogram, only $\mathrm{pN}$ and $\mathrm{pT}$ are potentially influenced by neoadjuvant therapy. We include cT in the calculation of the NAR score based on our belief that the degree of tumor downstaging is more important than the absolute pT. The remaining factors from the Valentini nomogram (age, gender, type of surgery, radiation dose, and receipt of adjuvant therapy) would not be influenced by neoadjuvant therapy and thus cannot contribute to a useful surrogate endpoint for assessing neoadjuvant treatment. The NAR formula, importantly, serves as a pseudo-continuous variable with 24 possible discrete scores from 0 to 100 with higher scores representing a poorer prognosis. The formula also standardizes downstaging by incorporating the treatment effect on the $\mathrm{T}$ stage, which accounts for bulky or large tumors regressing, but not to the point of ypCR. The relative weights of 5 for $\mathrm{pN}$ and 3 for downstaging of $\mathrm{T}$ were suggested by the Valentini nomogram and reflect the relative importance of these variables. The constant 12 assures that all scores are positive inside the brackets. Squaring the numerator transforms the score to a more uniform measure per unit change. The scaling factor 9.61 in the denominator ensures that the final scores range from 0 to 100 . The NAR score is meant to be used in clinical trials as a surrogate endpoint for survival. The score is designed to be particularly sensitive to changes in factors that are affected by neoadjuvant therapy. Changes in mean NAR scores between treatment arms as a result of intervention should translate to changes in OS. Importantly, the score uses both clinical and pathologic factors that are universally available in rectal cancer clinical trials, obviating the need

Table 2 Summary of major tumor regression grade systems in use

\begin{tabular}{lllll}
\hline Score & Dworak, et al. (score 0-4) [23] & $\begin{array}{l}\text { American Joint Committee } \\
\text { on Cancer (score 0-3) [26] }\end{array}$ & Mandard, et al. (score 1-5) [27] & $\begin{array}{l}\text { Memorial Sloan } \\
\text { Kettering } \\
\text { CC (score 1-3) [29] }\end{array}$ \\
\hline TRG 0 & Minimal tumor response to treatment & No residual tumor cells & - & - \\
TRG 1 & Fibrosis in $<25 \%$ of tumor & Single or small group of cells & No residual tumor cells & No residual tumor cells \\
TRG 2 & Fibrosis in 25-50 \% of tumor & Cancer with fibrotic response & Rare cancer cells & $86-99 \%$ tumor response \\
TRG 3 & Fibrosis in $>50 \%$ of tumor & Minimal tumor response to treatment & Fibrosis $>$ residual cancer & $\leq 85 \%$ tumor response \\
TRG 4 & No residual tumor cells & - & Residual cancer $>$ fibrosis & - \\
TRG 5 & - & - & Minimal tumor response to treatment -
\end{tabular}

$T R G$ tumor regression grade 


$$
N A R=\frac{[5 p N-3(c T-p T)+12]^{2}}{9.61}
$$

Fig. 1 Calculation of the neoadjuvant rectal (NAR) score. $c T$ is an element of the set $\{1,2,3,4\}, p T$ is in $\{0,1,2,3,4\}$, and $p N$ is in $\{0,1,2\}$. $c T$ clinical tumor stage, $p T$ pathologic tumor stage, $p N$ pathologic nodal stage

for additional trial infrastructure, cost, time, or effort. The score was not designed or intended for individual patient use or to provide prognosis as part of clinical care. In that regard, the original Valentini nomogram is better suited for that purpose.

After establishing the NAR score calculation, it was validated using the NSABP R-04 clinical trial patient dataset $[44 \bullet \bullet$. The NSABP R-04 study involved 1479 patients with stages II or III rectal cancer and randomized them to one of four neoadjuvant CRT arms testing different radiosensitizers including (1) continuous infusion 5-fluorouracil, (2) continuous infusion 5-fluorouracil with weekly oxaliplatin, (3) oral daily capecitabine, or (4) oral daily capecitabine plus weekly oxaliplatin. Outcomes were analyzed in a $2 \times 2$ factorial design to assess the relative differences between infusional 5-FU vs. capecitabine and oxaliplatin vs. no oxaliplatin [45]. Continuous NAR score was significantly associated with OS (HR/unit 1.04; $95 \%$ CI $1.03-1.05 ; p<0.0001)[44 \bullet \cdot]$. NAR scores in the NSABP R-04 trial dataset were categorized as low (NAR $<8)$, intermediate $(\mathrm{NAR}=8-16)$, and high $(\mathrm{NAR}>16)$ based on tertiles of the observed scores. These categories were significantly associated with OS $(p<0.0001)$ with 5 year OS values of 92,89 , and $68 \%$, respectively (Fig. 2). OS was also predicted by ypCR in this analysis, but continuous NAR score had a stronger association as measured by Akaike's information criterion $(p<0.0001)$. The NAR score was subsequently and independently further validated in an international clinical trial dataset providing further evidence of utility as a short-term surrogate [46].

\section{Conclusions and Future Directions}

The NAR score represents the next logical step in defining a short-term surrogate clinical trial endpoint in rectal cancer study design. It is undergoing trial level validation currently and otherwise meets all the requirements for a surrogate endpoint outlined in Table 1. The NAR score is now poised to serve as the primary endpoint of upcoming phase II studies being designed within NRG Oncology and the NCI National Clinical Trials Network. It has already been adopted as a secondary endpoint by several ongoing phase I and II studies testing novel radiosensitizers and other neoadjuvant interventions in rectal cancer, including the incorporation of induction chemotherapy or total neoadjuvant therapy. With the development of this surrogate endpoint and incorporation into rectal cancer clinical trials, we anticipate more rapid scientific progress to the benefit of our patients and their families.
Fig. 2 Overall survival by neoadjuvant rectal (NAR) score by group in NSABP R-04 clinical trial

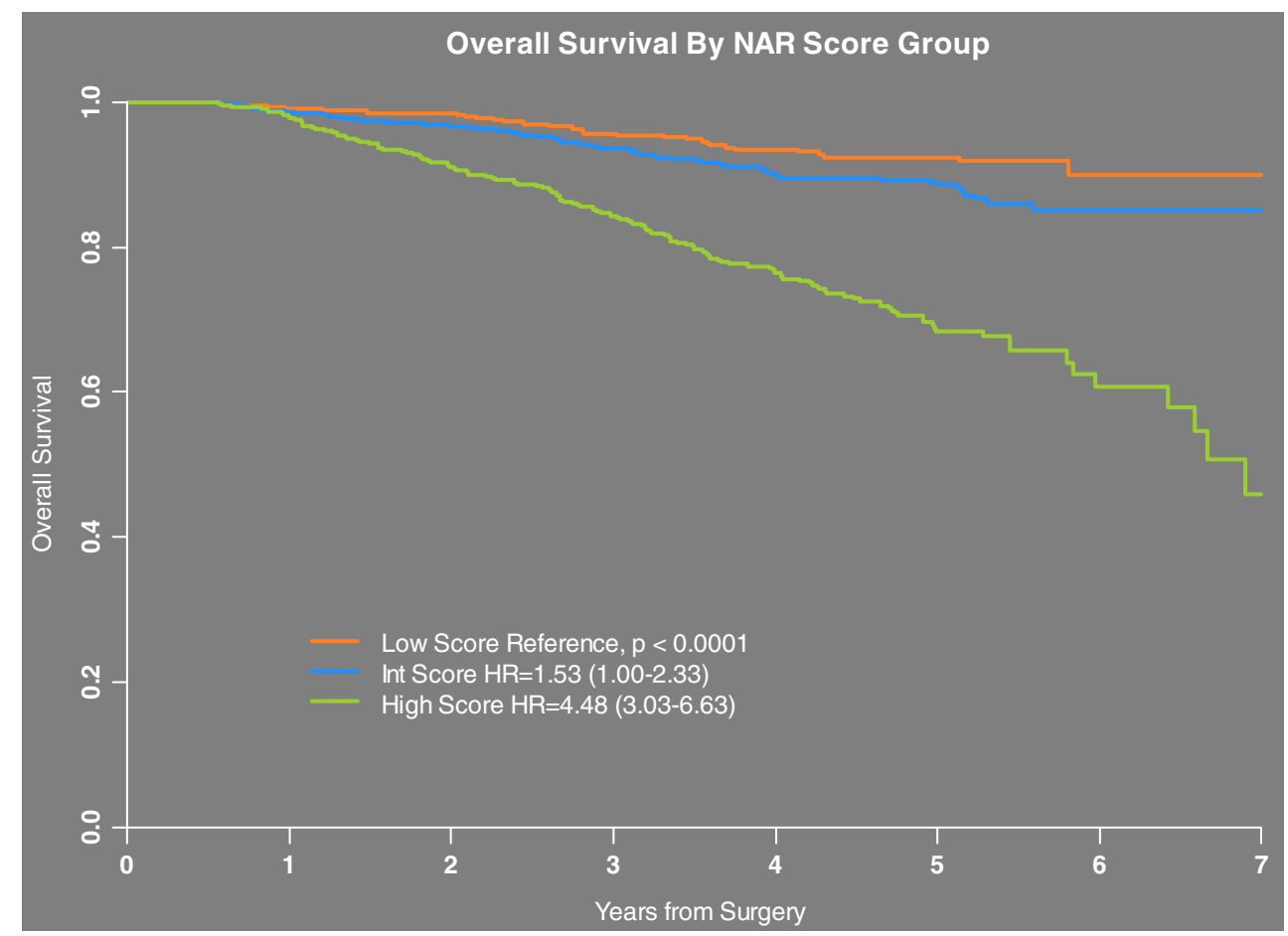




\section{Compliance with Ethics Guidelines}

Conflict of Interest The authors declare that they have no competing interests.

Human and Animal Rights and Informed Consent This manuscript does contain data from a clinical trial, NSABP R-04, which did involve research performed on human subjects. This trial was approved by the $\mathrm{NCI}$ and local IRBs. All patients provided their written informed consent prior to their treatment.

Open Access This article is distributed under the terms of the Creative Commons Attribution 4.0 International License (http:// creativecommons.org/licenses/by/4.0/), which permits unrestricted use, distribution, and reproduction in any medium, provided you give appropriate credit to the original author(s) and the source, provide a link to the Creative Commons license, and indicate if changes were made.

\section{References}

Papers of particular interest, published recently, have been highlighted as:

- Of importance

•- Of major importance

1. Howlader N, Noone AM, Krapcho M, Garshell J, Miller D, Altekruse SF, Kosary CL, Yu M, Ruhl J, Tatalovich Z, Mariotto A, Lewis DR, Chen HS, Feuer EJ, Cronin KA (eds). SEER Cancer Statistics Review, 1975-2012, National Cancer Institute. Bethesda, MD, http://seer.cancer.gov/csr/1975_2012/, based on November 2014 SEER data submission, posted to the SEER web site, April 2015.

2. Meester RGS, Doubeni CA, Zauber AG, Goede SL, Levin TR, Corley DA, et al. Public health impact of achieving $80 \%$ colorectal cancer screening rates in the United States by 2018. Cancer. 2015;121:2281-5. Predictive US Public Health analysis based upon mathematical modeling of what the total value to society and the healthcare system would be if colorectal cancer screening was improved to $80 \%$ of recommendations.

3. American Cancer Society. Cancer facts \& figures 2015. Atlanta: American Cancer Society; 2015.

4.• Cancer Genome Atlas Network. Comprehensive molecular characterization of human colon and rectal cancer. Nature. 2012;487(7407):330-7. Results of a global effort to generate a comprehensive molecular analysis of colorectal cancer revealing consistencies and opportunities for therapeutic intervention.

5. NCCN Clinical Practice Guidelines in Oncology ${ }^{\mathrm{TM}}$ (Rectal Cancer). V.3.2015. Accessed from the National Comprehensive Cancer Network website <http:/www.nccn.org/professionals/ physician_gls/PDF/rectal.pdf $>$ on June 20, 2015.

6. Gunderson LL, Jessup JM, Sargent DJ, Greene FL, Stewart A. Revised tumor and node categorization for rectal cancer based on surveillance, epidemiology, and end results and rectal pooled analysis outcomes. J Clin Oncol. 2010;28(2):256-63.

7.• Sauer R, Becker H, Hohenberger W, Rödel C, Wittekind C, Fietkau $\mathrm{R}$, et al. Preoperative versus postoperative chemoradiotherapy for rectal cancer. N Engl J Med. 2004;351(17):1731-40. Landmark clinical trial which helped to establish neoadjuvant chemoradiotherapy as a standard of care for rectal cancer. Results demonstrated improved compliance, reduced toxicity and no detriment in surgical outcomes or added risks with this approach.
8. Bosset JF, Collette L, Calais G, Mineur L, Maingon P, RadosevicJelic L, et al. Chemotherapy with preoperative radiotherapy in rectal cancer. N Engl J Med. 2006;355(11):1114-23.

9.• Roh MS, Colangelo LH, O'Connell MJ, Yothers G, Deutsch M, Allegra CJ, et al. Preoperative multimodality therapy improves disease-free survival in patients with carcinoma of the rectum: NSABP R-03. J Clin Oncol. 2009;27(31):5124-30. Another landmark clinical trial which helped to establish neoadjuvant chemoradiotherapy as a standard of care for rectal cancer. Results demonstrated improved compliance, reduced toxicity and no detriment in surgical outcomes or added risks with this approach.

10. Prentice RL. Surrogate endpoints in clinical trials: definition and operational criteria. Stat Med. 1989;8(4):431-40.

11. De Gruttola VG, Clax P, Demets DL, Downing GJ, Ellenberg SS, Friedman L, et al. Considerations in the evaluation of surrogate endpoints in clinical trials. Summary of a National Institutes of Health workshop. Control Clin Trials. 2001;22(5):485-502.

12. Buyse M, Molenberghs G. Criteria for the validation of surrogate endpoints in randomized experiments. Biometrics. 1998;54(3): 1014-29.

13. Köckerling F, Reymond MA, Altendorf-Hofmann A, Dworak $\mathrm{O}$, Hohenberger W. Influence of surgery on metachronous distant metastases and survival in rectal cancer. J Clin Oncol. 1998;16(1):324-9.

14. Glynne-Jones R, Mawdsley S, Pearce T, Buyse M. Alternative clinical end points in rectal cancer-are we getting closer? Ann Oncol. 2006;17(8):1239-48.

15. van der Pas MH, Meijer S, Hoekstra OS, Riphagen II, de Vet HC, $\mathrm{Knol} \mathrm{DL}$, et al. Sentinel-lymph-node procedure in colon and rectal cancer: a systematic review and meta-analysis. Lancet Oncol. 2011;12(6):540-50.

16. Glynne-Jones R, Wallace M, Livingstone JI, Meyrick-Thomas J. Complete clinical response after preoperative chemoradiation in rectal cancer: is a "wait and see" policy justified? Dis Colon Rectum. 2008;51(1):10-9.

17. Washington MK, Berlin J, Branton P, Burgart LJ, Carter DK, Fitzgibbons PL, et al. Protocol for the examination of specimens from patients with primary carcinoma of the colon and rectum. Arch Pathol Lab Med. 2009;133(10):1539-51.

18. Quirke P, Durdey P, Dixon MF, Williams NS. Local recurrence of rectal adenocarcinoma due to inadequate surgical resection. Histopathological study of lateral tumour spread and surgical excision. Lancet. 1986;2(8514):996-9.

19. Birbeck KF, Macklin CP, Tiffin NJ, Parsons W, Dixon MF, Mapstone NP, et al. Rates of circumferential resection margin involvement vary between surgeons and predict outcomes in rectal cancer surgery. Ann Surg. 2002;235(4):449-57.

20. Maas M, Nelemans PJ, Valentini V, Das P, Rödel C, Kuo LJ, et al. Long-term outcome in patients with a pathological complete response after chemoradiation for rectal cancer: a pooled analysis of individual patient data. Lancet Oncol. 2010;11(9):835-44.

21. Chua YJ. Pathological complete response: still a relevant endpoint in rectal cancer? Lancet Oncol. 2010;11(9):807-8.

22. Das P, Skibber JM, Rodriguez-Bigas MA, Feig BW, Chang GJ, Wolff RA, et al. Predictors of tumor response and downstaging in patients who receive preoperative chemoradiation for rectal cancer. Cancer. 2007;109(9):1750-5.

23. Dworak O, Keilholz L, Hoffmann A. Pathological features of rectal cancer after preoperative radiochemotherapy. Int J Color Dis. 1997;12(1):19-23.

24. Trakarnsanga A, Gönen M, Shia J, Nash GM, Temple LK, Guillem JG, Paty PB, Goodman KA, Wu A, Gollub M, Segal N, Saltz L, Garcia-Aguilar J, Weiser MR. Comparison of tumor regression grade systems for locally advanced rectal cancer after multimodality treatment. J Natl Cancer Inst. 2014 Sep 22;106(10). Valuable and 
unbiased comparison of all major tumor regression grade systems in current use for rectal cancer. Limitations and recommendations are included.

25. Minsky BD, Rodel C. Identifying the most predictive postchemoradiation TRG system for rectal cancer. J Natl Cancer Inst. 2014 Sep 22;106(10). Outstanding editorial associated with reference \#24 detailing how to move past the need for central pathology review for tumor regression grading for rectal cancers.

26. Mace AG, Pai RK, Stocchi L, Kalady MF. American Joint Committee on Cancer and College of American Pathologists regression grade: a new prognostic factor in rectal cancer. Dis Colon Rectum. 2015;58(1):32-44.

27. Mandard AM, Dalibard F, Mandard JC, Marnay J, Henry-Amar M, Petiot JF, et al. Pathologic assessment of tumor regression after preoperative chemoradiotherapy of esophageal carcinoma. Clinico-Pathologic Correlations Cancer. 1994;73:2680-6.

28. Rödel C, Martus P, Papadoupolos T, Füzesi L, Klimpfinger M, Fietkau R, et al. Prognostic significance of tumor regression after preoperative chemoradiotherapy for rectal cancer. J Clin Oncol. 2005;23(34):8688-96.

29. Quah HM, Chou JF, Gonen M, Shia J, Schrag D, Saltz LB, et al. Pathologic stage is most prognostic of disease-free survival in locally advanced rectal cancer patients after preoperative chemoradiation. Cancer. 2008;113(1):57-64.

30. Agarwal A, Chang GJ, Hu CY, Taggart M, Rashid A, Park IJ, et al. Quantified pathologic response assessed as residual tumor burden is a predictor of recurrence-free survival in patients with rectal cancer who undergo resection after neoadjuvant chemoradiotherapy. Cancer. 2013;119:4231-41.

31. Park IJ, You YN, Agarwal A, Skibber JM, Rodriguez-Bigas MA, Eng $\mathrm{C}$, et al. Neoadjuvant treatment response as an early response indicator for patients with rectal cancer. J Clin Oncol. 2012;30(15):1770-6.

32. Fokas E, Liersch T, Fietkau R, Hohenberger W, Beissbarth T, Hess $\mathrm{C}$, et al. Tumor regression grading after preoperative chemoradiotherapy for locally advanced rectal carcinoma revisited: updated results of the CAO/ARO/AIO-94 trial. J Clin Oncol. 2014;32(15): 1554-62.

33. Huebner M, Wolff BG, Smyrk TC, Aakre J, Larson DW. Partial pathologic response and nodal status as most significant prognostic factors for advanced rectal cancer treated with preoperative chemoradiotherapy. World J Surg. 2012;36(3):675-83.

34. Birlik B, Obuz F, Elibol FD, Celik AO, Sokmen S, Terzi C, et al. Diffusion-weighted MRI and MR-volumetry - in the evaluation of tumor response after preoperative chemoradiotherapy in patients with locally advanced rectal cancer. Magn Reson Imaging. 2015;33(2):201-12.

35. Altini C, Niccoli Asabella A, De Luca R, Fanelli M, Caliandro C, Quartuccio N, et al. Comparison of (18)F-FDG PET/CT methods of analysis for predicting response to neoadjuvant chemoradiation therapy in patients with locally advanced low rectal cancer. Abdom Imaging. 2015;40(5):1190-202.

36. Li C, Lan X, Yuan H, Feng H, Xia X, Zhang Y. 18F-FDG PET predicts pathological response to preoperative chemoradiotherapy in patients with primary rectal cancer: a meta-analysis. Ann Nucl Med. 2014;28(5):436-46.
37. Genovesi D, Filippone A, Ausili Cèfaro G, Trignani M, Vinciguerra A, Augurio A, et al. Diffusion-weighted magnetic resonance for prediction of response after neoadjuvant chemoradiation therapy for locally advanced rectal cancer: preliminary results of a monoinstitutional prospective study. Eur J Surg Oncol. 2013;39(10):1071-8.

38. Patel UB, Brown G, Rutten H, West N, Sebag-Montefiore D, Glynne-Jones R, et al. Comparison of magnetic resonance imaging and histopathological response to chemoradiotherapy in locally advanced rectal cancer. Ann Surg Oncol. 2012;19(9):2842-52.

39. Patel UB, Taylor F, Blomqvist L, George C, Evans H, Tekkis P, et al. Magnetic resonance imaging-detected tumor response for locally advanced rectal cancer predicts survival outcomes: MERCURY experience. J Clin Oncol. 2011;29(28):3753-60.

40.• Valentini V, van Stiphout RG, Lammering G, Gambacorta MA, Barba MC, Bebenek M, et al. Nomograms for predicting local recurrence, distant metastases, and overall survival for patients with locally advanced rectal cancer on the basis of European randomized clinical trials. J Clin Oncol. 2011;29(23):3163-72. Sophisticated and validated nomograms for predicting local recurrence, distant mets and overall survival for rectal cancer patients treated in select RCTs. Serves, in part, as the basis for the NAR Score.

41. Gérard JP, Conroy T, Bonnetain F, Bouché O, Chapet O, ClosonDejardin MT, et al. Preoperative radiotherapy with or without concurrent fluorouracil and leucovorin in T3-4 rectal cancers: results of FFCD 9203. J Clin Oncol. 2006;24(28):4620-5.

42. Bujko K, Nowacki MP, Kepka L, Oledzki J, Bebenek M, Kryj M, et al. Postoperative complications in patients irradiated preoperatively for rectal cancer: report of a randomised trial comparing short-term radiotherapy vs chemoradiation. Color Dis. 2005;7(4): $410-6$.

43. Sainato A, Cernusco Luna Nunzia V, Valentini V, De Paoli A, Maurizi ER, Lupattelli M, et al. No benefit of adjuvant Fluorouracil Leucovorin chemotherapy after neoadjuvant chemoradiotherapy in locally advanced cancer of the rectum (LARC): long term results of a randomized trial (I-CNR-RT). Radiother Oncol. 2014;113(2):223-9.

44.• Yothers G, George TJ, Petrelli NJ, O'Connell MJ, Beart RW, Allegra CJ, Roh MS, Lopa S, Sharif S, Wolmark N. Neoadjuvant rectal cancer (RC) score predicts survival: potential surrogate endpoint for early phase trials. J Clin Oncol 32:5s, 2014 (suppl; abstr 3533). International podium presentation at the ASCO Annual Meeting where the NAR Score was debuted with the associated data to support the validation.

45. O'Connell MJ, Colangelo LH, Beart RW, Petrelli NJ, Allegra CJ, Sharif S, et al. Capecitabine and oxaliplatin in the preoperative multimodality treatment of rectal cancer: surgical end points from National Surgical Adjuvant Breast and Bowel Project trial R-04. J Clin Oncol. 2014;32(18):1927-34.

46. Raissouni S, Mercer J, Gresham G, Kumar A, Goodwin RA, Jiang M, Leung A, Heng D, Tang PA, Doll CM, MacLean A, Powell ED, Hiller JP, Monzon JG, Cheung WJ, Vickers MM. External validation of the neoadjuvant rectal (NAR) score and Valentini prediction nomogram (VPN): a multicenter study. J Clin Oncol 32:5s, 2014 (suppl; abstr 3532). 